\title{
EL PORTAFOLIO COMO INSTRUMENTO CLAVE PARA LA EVALUACIÓN EN EDUCACIÓN SUPERIOR
}

THE PORTFOLIO AS A KEY TOOL FOR ASSESSMENT IN HIGHER EDUCATION

\author{
Volumen 12, Número 1 \\ Enero-Abril
}

pp. $1-23$

Este número se publicó el 28 de febrero de 2012

Gabriela Murillo Sancho

Revista indizada en REDALYC

Revista distribuida en las bases de datos:

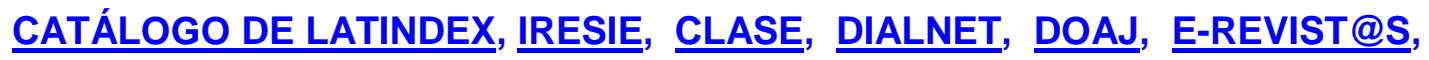

Revista registrada en los directorios:

ULRICH'S, REDIE, RINACE, OEI, MAESTROTECA, PREAL, HUASCARAN, CLASCO 


\title{
EL PORTAFOLIO COMO INSTRUMENTO CLAVE PARA LA EVALUACIÓN EN EDUCACIÓN SUPERIOR \\ THE PORTFOLIO AS A KEY TOOL FOR ASSESSMENT IN HIGHER EDUCATION
}

\author{
Gabriela Murillo Sancho ${ }^{1}$
}

\begin{abstract}
Resumen: El ensayo presenta una descripción del portafolio como herramienta para evaluar, tanto desempeños docentes como estudiantiles en Educación Superior, además de una reflexión sobre su importancia para la evaluación de docentes y estudiantes. El escrito desarrolla una visión particular sobre el portafolio, su constitución y usos, enfocándose en su valor en el marco de la evaluación. Se elabora como un aporte para la reflexión y las ideas en didáctica evaluativa y, por ende, para el mejoramiento de la docencia universitaria. Se incluye una rúbrica como propuesta para calificar el portafolio. La conclusión principal radica en el rol del portafolio para la evaluación universitaria de docentes y estudiantes.
\end{abstract}

Palabras clave: DOCENCIA UNIVERSITARIA, EVALUACIÓN, PORTAFOLIO

\begin{abstract}
This paper presents a description of the portfolio as a tool to evaluate teachers and students performances in higher education. Besides, it presents a reflection about the importance of portfolios in teachers and students evaluation. The document develops a particular vision about the portfolio, its constitution and uses, focusing in its value in the outline of the evaluation. It is elaborated as a contribution to the reflection and the ideas about evaluative didactics and consequently, for the improvement of the university teaching. A rubric is included as a proposal to evaluate the portfolio. The main conclusion consists in the role of the portfolio for the evaluation of university teachers and students.
\end{abstract}

Key words: TEACHING OF UNIVERSITY, EVALUATION, PORTFOLIO

\footnotetext{
${ }^{1}$ Magister en Psicopedagogía de la Universidad Estatal a Distancia, Costa Rica y Licenciada en Educación Primaria, de la Universidad de Costa Rica. Investigadora del Instituto de Investigación en Educación, Docente en la Escuela de Salud Pública y en el Postgrado en Enfermería de la Universidad de Costa Rica. Apoya en la Sección Técnica de Evaluación, STEA del Centro de Evaluación Académica de la Universidad de Costa Rica.
}

Dirección electrónica: maria.murillo@ucr.ac.cr

Artículo recibido: 16 de setiembre, 2011

Aprobado: 26 de enero, 2012 


\section{Introducción}

En primera instancia, es importante señalar la concepción del objeto de este escrito, el portafolio. De manera elemental, se considera como un espacio físico, digital y/o virtual que sirve para recopilar trabajos, escritos diversos, fotografías y cualquier otro elemento que señale algún registro que se desea realizar. De forma más compleja, el portafolio toma como base la idea anterior, pero tendría, además, una intención de aprendizaje o didáctica, especificada en objetivos y reflejada en el hilo conductor de su desarrollo.

Dos roles principales se ejercen en el desarrollo del portafolio como herramienta, uno es el de sujeto que asume su aprendizaje y muestra sus desempeños y avances durante el proceso de construcción y finalización del portafolio -para efectos de Educación Superior puede ser un docente o un estudiante-. El otro rol es el de evaluador, persona que dará seguimiento al proceso de desarrollo del portafolio y contrastará los avances respecto de los criterios delimitados para los niveles de logro esperados, asunto que se presentará más adelante.

Al respecto y de acuerdo con lo propuesto por Argudín (2007), para efectos del desarrollo de un portafolio, la persona que lo realiza puede participar en la selección de los contenidos, en la organización del proceso y de la herramienta, así como en la delimitación de los criterios valorativos y de las evidencias esperadas para los procesos realizados. También, puede integrarse en la evaluación de su propio trabajo. Complementariamente, quien evalúa un portafolio tiene la posibilidad de elaborar un registro sobre el progreso del que lo desarrolla y aportar con esto a las bases utilizadas para valorar distintos aspectos que se consideren relevantes del mismo.

Además, puede hablarse de tipos de portafolios, los cuales serán considerados según su naturaleza y finalidad, de la siguiente manera: creativo, escolar, de diseño, de evidencias, de servicios, de productos o resultados, entre otros. La clase de portafolio que se decida hacer, también puede asumir uno o combinar diversos formatos tal como el físico (material), el digital o el electrónico.

Para efectos de este escrito, el portafolio constituye entonces una herramienta educativa. Su potencial es muy amplio y de ahí el uso expansivo y diverso en ámbitos tales como: educación formal (primaria, secundaria, superior), arte, fotografía, arquitectura, diseño, otros. Dada esta finalidad educativa, es importante dar seguimiento a las personas mientras lo elaboran, enfatizando en sus propios progresos al desarrollarlo; estimularlas para que no 
se conformen con resultados de apariencia (cantidad de cosas registradas, por ejemplo), sino que se dirijan hacia logros significativos; resaltar el desarrollo personal y colectivo e intentar integrar los conocimientos previos en cada situación-problema que se aborda; destacar logros que impulsan el desarrollo como personas; resolver problemas con base en información sólida y procesos claros y enfocados, son algunos de los objetivos que pueden asignarse a la construcción de un portafolio.

Mejorar los propios desempeños utilizando una herramienta como esta, resulta ser el principal desafío de los procesos evaluativos que se realicen a través del portafolio.

\section{Desarrollo del tema}

De acuerdo con Hernández (2006), el portafolio constituye un método de enseñanza, y también de evaluación, el cual básicamente consiste en el aporte de producciones de diferente índole por parte de quien es docente o de quien es estudiante. Es a través de estas evidencias que se pueden valorar los desempeños en el marco de una disciplina o curso específico. Dichas elaboraciones dan cuenta del proceso personal seguido por quien elabora el portafolio, ya que reflejan esfuerzos, dificultades, logros y propuestas alternativas, en relación con los objetivos y los criterios de evaluación establecidos previamente, así como con los rasgos de competencia y los resultados esperados, según el caso.

En cuanto modelo particular para el registro de evidencias, el portafolio se fundamenta en la idea de que la evaluación muestra la manera en cómo una persona que aprende organiza su aprendizaje, o sea, testimonia de alguna manera su estilo para aprender. Además, responde, entre otros, a dos aspectos fundamentales: a) un procedimiento metodológico para su desarrollo que incluye las técnicas y actividades didácticas realizadas en los procesos formativos; $y, b$ ) el procedimiento valorativo por el que se articula el conjunto de evidencias en la formación de los rasgos de competencias o de objetivos esperados. Si se usaran otros instrumentos de evaluación más tradicionales que aportan una visión más fragmentada, esto sería más difícil de registrar.

Según señala Argudín (2007) y de acuerdo con lo vivido, existen distintos tipos de portafolios, los cuales responden a intencionalidades de quien lo realiza y de quien lo haya solicitado (docentes para el caso de estudiantes, pares para el caso de docentes). Se toma en cuenta el formato utilizado -como ya se ha mencionado- y también el contenido. De acuerdo con esto último, se dan ciertas clasificaciones, las cuales aquí no pretenden ser 
exhaustivas ni excluyentes, sino permitir la identificación de los diversos tipos y sus posibles combinaciones. A continuación, se señalan algunas, a manera de ejemplo:

\section{Los portafolios de habilidades}

Con la forma de recopilar la información y de acuerdo con el seguimiento que reciba, quien lo realiza puede mostrar el proceso formativo llevado adelante y también reconocer las destrezas que ha desarrollado.

2. Portafolios para desarrollar los curriculum personales o historias de vida.

Contiene básicamente la historia académica de la persona. Incluye actividades formales y extracurriculares, todas ellas con algún significado e importancia en la vida profesional.

Este tipo de portafolios ayuda a las personas a identificar las capacidades que ha desarrollado y los aprendizajes que ha construido y cómo puede utilizarlos; facilita el distinguir aquellos que son significativos y el organizarlos respecto del propio ámbito laboral. Puede incluir constancias académicas, reconocimientos, ejemplos de trabajos realizados; cursos realizados, resultados particulares de algunas acciones significativas, cartas de recomendación, otros. El portafolio podrá organizarse de la misma manera en que presentará los documentos en el ámbito laboral.

\section{Portafolios para un curso}

Las secciones del portafolio se delimitarán, por ejemplo, según la propuesta de desarrollo del curso, o de acuerdo con los temas a tratar; la organización puede estar dada por el docente o realizada por el mismo estudiante. El apoyo, en término de seguimiento, constituye un elemento importante en este tipo de portafolios.

\section{Portafolio tipo vitrina}

Contiene evidencia limitada y resulta útil en laboratorios. Por ejemplo, mostrar el mejor trabajo, el trabajo mejorado, el peor trabajo, el trabajo preferido. Los anexos pueden ser tareas, exámenes, otros. 


\section{Portafolios de cotejo}

Contiene un número predeterminado de anexos. Por ejemplo, se solicita documentar el portafolio con diez problemas y dos resúmenes realizados bajo criterios específicos, un artículo, dos reportes y dos exámenes a los que anexe sus reflexiones.

\section{Portafolios formato abierto}

Incluyen lo que su creador considere como evidencia de su aprendizaje. Se requiere delimitar aspectos sobre su elaboración y evaluación.

\section{Portafolios docentes}

Muestran las evidencias de los desempeños docentes. Recopilan información personal, del curso, de sus actividades académicas y todas aquellas evidencias que constituyan una manifestación de su quehacer, de su crecimiento, de sus propuestas de mejoramiento.

En cualquier tipo de portafolio, la persona que lo realiza tiene la responsabilidad del desarrollo de las fases, así como la demostración de organización, iniciativa y creatividad en su construcción. Para Fernández y Maiqués (2001), la elaboración de un portafolio supone todo un giro metodológico en relación con los modelos anteriores de análisis o evaluación de desempeños docentes y estudiantiles.

Con base en la propia experiencia y de acuerdo con los autores consultados, entre los méritos o ventajas que se obtienen al desarrollar un portafolio pueden señalarse algunas tales como:

$\checkmark$ Impulsa la autonomía de quien lo realiza.

$\checkmark$ Favorece el pensamiento crítico y reflexivo lo que garantiza resultados mínimos o básicos de aprendizaje.

$\checkmark$ Permite y promociona la formación de competencias.

$\checkmark$ Ofrece información amplia sobre los aspectos que se están formando.

$\checkmark$ Se constituye en una fuente de evaluación continua.

$\checkmark$ Desde el principio cuenta con los criterios de evaluación respectivos, según lo esperado en cuanto a desempeños y evidencias.

$\checkmark$ Muestra un carácter cooperativo, en los casos donde participan dos o más personas en su desarrollo: por ejemplo el docente y el estudiante. 
$\checkmark$ Al desarrollar esta estrategia, la persona proyecta la diversidad de evidencias de desempeños que ha ido haciendo propios.

$\checkmark$ Se identifican situaciones positivas y también problemáticas, así como las alternativas para resolverlas y/o mejorar las condiciones de desempeño.

$\checkmark$ Los resultados se pueden compartir con otros compañeros y con otros docentes.

$\checkmark$ Favorece hábitos cognitivos y sociales, tales como la síntesis, el análisis, la conceptualización, el compartir hallazgos, la consulta y los comentarios, la formación de opinión propia, otros.

$\checkmark$ Conlleva un componente motivador y de estímulo para quien lo realiza, al tratarse de un trabajo continuado donde se van comprobando rápidamente los esfuerzos y resultados conseguidos.

$\checkmark$ Impulsa también al mejoramiento inmediato, alejándose de una visión tradicional de evaluación (sumativa final) y trascendiendo hacia la evaluación de proceso con acciones de cambio, de transformación y de propuesta, en el momento que se requiera.

$\checkmark$ Favorece la consecución de metas personales y del curso (o actividad para la que se propuso) con niveles diferentes y con temporalidades también adaptadas a cada situación de avance personal.

$\checkmark$ Es una construcción-producción personalizada, por lo que no hay dos iguales.

$\checkmark$ Promueve la participación en el monitoreo y evaluación del propio aprendizaje.

$\checkmark$ Propicia la asunción de la responsabilidad en el propio aprendizaje.

$\checkmark$ Brinda la oportunidad de dar a conocer actitudes, intereses, valores.

$\checkmark$ Provee información diversa que puede resultar valiosa respecto de ciertos aspectos del proceso de aprendizaje personal.

$\checkmark$ Los docentes pueden cotejar algunas de sus intenciones didácticas al observar las evidencias y avances.

$\checkmark$ Se puede adaptar a diversas necesidades, intereses y capacidades de quien lo realiza.

$\checkmark$ Promueve la autoevaluación.

$\checkmark$ Permite seleccionar a estudiantes o docentes para tareas o acciones requeridas, de acuerdo a los desempeños mostrados.

$\checkmark$ Permite contar con una visión más amplia de lo que la persona ha aprendido.

$\checkmark$ Otorga mayor responsabilidad a su creador de mostrar cómo aprende, cómo avanza. 
Complementariamente, algunas situaciones relacionadas con el diseño y desarrollo del portafolio podrían convertirse en dificultades u obstáculos, según la perspectiva con que se asuma el reto de llevarlo adelante:

$\checkmark$ Alto grado de compromiso, autodisciplina y responsabilidad que se requiere por parte de quien desarrolla el portafolio.

$\checkmark$ Dudas que surgen al pensar que algo no se está haciendo como se debería.

$\checkmark$ Pérdida de tiempo por parte del docente y del estudiante, si no se organiza su desarrollo y su correspondiente evaluación.

$\checkmark$ Posibles contradicciones internas en el curso y en el desempeño docente si no se maneja de forma integral y coherente respecto a otros tipos de instrumentos de evaluación didáctica tales como exámenes, quices, reportes, otros.

$\checkmark$ Utilizar el portafolio implica un estilo de docencia y de aprendizaje distante de los tradicionales.

$\checkmark$ Sistematización de los resultados de la evaluación en función de los objetivos y/o las competencias, y complementariamente implementación de las acciones de mejora necesarias para atender las situaciones emergentes.

$\checkmark$ Exige la dedicación de mucho tiempo para su desarrollo y evaluación.

$\checkmark$ Requiere un claro y específico proceso de evaluación.

$\checkmark$ Difícilmente generaliza resultados.

$\checkmark$ No mide específicamente el nivel de conocimiento como lo hace otro tipo de instrumentos de evaluación.

$\checkmark$ Puede prestarse para prácticas deshonestas (copia, plagio, etc.) dado que su elaboración no necesariamente es controlada en un salón de clase, por ejemplo.

En la literatura consultada para la elaboración de este escrito y también de acuerdo con la experiencia, se puede afirmar que existe una estructura variada para el diseño del portafolio, la cual dependerá de los objetivos y finalidad por la que se plantee el portafolio y también de acuerdo con la particularidad y recursos de quien lo desarrolle. Sin embargo, se pueden diferenciar algunos apartados que sería importante tomar en cuenta al respecto:

1. Presentación que incluirá el nombre de la persona que realiza el portafolio, de la instancia u organización marco de su desarrollo, algún signo relacionado con esta presentación inicial u otro detalle que se considere relevante en término de identificación. 
2. Guía o un índice de contenidos que incluirá las dimensiones que serán desarrolladas, determinará el tipo de trabajo y estrategia didáctica. Este apartado puede estar totalmente delimitado por una persona o instancia externa, o más abierto a una dirección por parte de quien lo desarrolla.

3. Apartado introductorio al portafolio que detallará los objetivos y/o competencias que se propone lograr/formar, así como las intenciones, creencias y punto de partida inicial de un tema o área determinada.

4. Cuerpo del portafolio, centrado en el desarrollo de los temas, núcleos centrales o dimensiones propuestos en el índice. Contiene la documentación seleccionada por quien realiza el portafolio, recopila evidencias de desempeños en cada aspecto y muestra la reflexión realizada en cada uno de ellos. Incluye los recursos básicos que serán utilizados; también, presenta las acciones valorativas y aquellas estratégicas y/o de mejora, ya sea para cada dimensión o recopiladas de manera organizada en un plan específico, llamado plan de mejora por algunas personas conocedoras de temas relacionados con autoevaluación.

5. Apartado de cierre como síntesis del aprendizaje en relación con la experiencia misma del portafolio y los desempeños sobre los que se recopilaron evidencias y se hicieron reflexiones.

Un ejemplo de estructura para un portafolio docente, de acuerdo con Fernández y Maiqués (2001), es la siguiente:

$\checkmark$ I. Nombre del profesor/a

$\checkmark$ II. Departamento/centro

$\checkmark$ III. Institución

$\checkmark$ IV. Fecha

$\checkmark$ V. Tabla de contenidos.

$\checkmark$ Responsabilidades de enseñanza.

$\checkmark$ Declaración de Filosofía de la enseñanza.

$\checkmark$ Metodología de la enseñanza. Estrategias. Objetivos.

$\checkmark$ Calificación de los estudiantes en los exámenes.

$\checkmark$ Evaluaciones de los colegas.

$\checkmark$ Declaración del jefe del departamento.

$\checkmark$ Detalle representativo del programa del curso. 
$\checkmark$ Productos de enseñanza (evidencia de lo que aprende el estudiante).

$\checkmark$ Premios y reconocimientos de su enseñanza.

$\checkmark$ Metas de enseñanza: a corto plazo y a largo plazo.

$\checkmark$ Apéndices.

Cualquier estructura que se decida resulta lineal en un formato físico (papel), pero puede ser versátil en un formato electrónico, digital, como veremos más adelante, de manera que exista la posibilidad de establecer conexiones entre los apartados y generar otros nuevos o algunas subdivisiones, según se decida el diseño del portafolio y se desarrolle su construcción personal.

Y también según escribe Barberá (2005), existe un cierto consenso entre los autores que han trabajado sobre este tema en relación con las FASES deseables para el desarrollo de un portafolio, a estas añadiremos algunas con base en la propia experiencia.

\section{Fase 1. Diseño: planificación del portafolio}

Idea general del trabajo que se desarrollará: su estructura, seguimiento, presentación, otros. Es medular la respuesta preliminar a dos asuntos: ¿qué se trabajará?, dimensiones, ejes, contenidos; ¿por qué?, las razones por las cuales se desarrollará el portafolio, la finalidad, el propósito, las metas, en fin, dependiendo del enfoque de la persona que lo desarrolla (docente o estudiante); asimismo, se establecerá la planificación general. Se decidirá sobre el formato, el tiempo de desarrollo del portafolio, las actividades que se articularán, y otros.

\section{Fase 2. Desarrollo:}

\subsection{Recolección de evidencias}

De acuerdo con la planificación, como formas de recopilación se consideran: a) documentos en diverso formato (digital, papel, vídeo, audio, otro), b) informaciones de diferentes tipos de contenido (conceptual, procedimental y actitudinal o normativo); c) tareas realizadas en clase o fuera de ella (mapas conceptuales, recortes varios, exámenes, informes, entrevistas, otros), d) otros. Estas evidencias vendrán determinadas por los objetivos y competencias formuladas para el portafolio. 


\subsection{Selección de evidencias}

En esta fase se han de elegir las pruebas más significativas respecto a los desempeños desarrollados y/o a los objetivos propuestos. Se trata de que muestren un desarrollo pertinente y creciente respecto de los procesos formativos que se pretenden evidenciar en el portafolio.

\subsection{Reflexión y análisis sobre los desempeños y las evidencias}

Desde cada uno de las dimensiones, ejes o contenidos definidos en el diseño del portafolio, y con base en las evidencias seleccionadas, se realizarán análisis y reflexiones críticas, constructivas, que muestren un nivel de profundidad creciente y particularmente una visión prospectiva del mejoramiento de la función docente y del aprendizaje del estudiante. Este proceso reflexivo, progresivo, es lo que algunas personas conocedoras de procesos para acreditación denominan "autoevaluación”.

\section{Fase 3. Evaluación:}

PROCESO: se ubica como fase en tanto elemento importante para el desarrollo del portafolio, pero no en cuanto a temporalidad estrictamente hablando. Se trata de pensar una evaluación continua, procesual y también final.

SUJETOS: se recomienda, por el éxito de la experiencia del uso de portafolio con estudiantes, que la persona que realiza su portafolio, efectúe una evaluación diagnóstica que apoye el diseño-planificación del portafolio: identificación de necesidades e intereses, delimitación de objetivos y competencias, etc. Complementariamente, es importante que el portafolio tenga una valoración externa. Si es el docente quien lo realiza, es recomendable que sean sus pares los que valoran y si es el estudiante, que valore el docente y también sus pares.

TÉCNICAS E INSTRUMENTOS: los procedimientos en término de técnicas y acciones evaluativas, serán definidos según el tipo y formato del portafolio, pero particularmente desde sus finalidades, según se hayan propuesto: objetivos o competencias.

Se recomienda la construcción de una matriz de evaluación, también llamada rúbrica, la cual se elabora con el fin de realizar una "evaluación objetiva y consistente", y determinar el nivel de dominio de una competencia, según anota Velásquez (2007). Esta matriz ha de ofrecerse a la persona que realiza el portafolio, de manera que tenga claridad de los 
aspectos, criterios y niveles que se tomarán en cuenta para valorar sus avances mediante el portafolio que está desarrollando. Con esta rúbrica, la persona que evalúa debe ajustarse a su contenido, de tal forma que la evaluación sea "justa e imparcial” (Velásquez, 2007).

Para diseñar una rúbrica, en concordancia con las ideas de este autor, se debe tomar en cuenta: a) las evidencias y los productos esperados, principalmente el portafolio como tal y complementariamente los avances que vaya presentando en su construcción; b) los aspectos a evaluar, en relación con los elementos de las evidencias y los indicadores de logro respecto de cada elemento; y finalmente, c) el nivel de logro de cada competencia, reflejados en una escala.

Como ejemplo de diseño de una matriz de evaluación o rúbrica tenemos:

\begin{tabular}{|c|c|c|c|c|c|c|}
\hline \multirow[b]{2}{*}{ Aspecto } & \multicolumn{6}{|c|}{ Nivel de desempeño } \\
\hline & $\begin{array}{c}\text { Creativo/ } \\
\text { propositivo (5) }\end{array}$ & Propositivo (4) & Autónomo (3) & Suficiente (2) & Deficiente (1) & $\begin{array}{c}\text { No } \\
\text { aceptable } \\
\text { (0) }\end{array}$ \\
\hline $\begin{array}{l}\text { 1. Bitácoras } \\
\text { de clases, } \\
\text { reuniones y } \\
\text { otras } \\
\text { actividades }\end{array}$ & $\begin{array}{l}\text { Registros } \\
\text { completos, } \\
\text { descriptivos; } \\
\text { incluyen } \\
\text { evidencias que } \\
\text { clarifican los } \\
\text { procesos, por ej: } \\
\text { fotos, collages, } \\
\text { otros; logros y } \\
\text { dificultades } \\
\text { claramente } \\
\text { identificados; } \\
\text { oportunidades y } \\
\text { propuestas de } \\
\text { mejora; reflexión } \\
\text { sobre lo anterior; } \\
\text { propuestas } \\
\text { concretas } \\
\text { respecto a lo } \\
\text { vivido en las } \\
\text { sesiones de las } \\
\text { que se elabora } \\
\text { bitácora. }\end{array}$ & $\begin{array}{l}\text { Registros } \\
\text { completos y } \\
\text { descriptivos; se } \\
\text { presentan } \\
\text { evidencias y } \\
\text { muestras de } \\
\text { algunas de ellas; } \\
\text { se mencionan } \\
\text { logros y } \\
\text { dificultades } \\
\text { relevantes; se } \\
\text { hacen algunas } \\
\text { propuestas de } \\
\text { mejoramiento. }\end{array}$ & $\begin{array}{l}\text { Registros } \\
\text { bastante } \\
\text { completos, } \\
\text { descriptivos; se } \\
\text { incluyen } \\
\text { algunas } \\
\text { evidencias y } \\
\text { algunos logros } \\
\text { y dificultades } \\
\text { presentados; } \\
\text { se menciona la } \\
\text { posibilidad de } \\
\text { mejora y se } \\
\text { establece a } \\
\text { nivel general, } \\
\text { cuál sería la } \\
\text { forma de } \\
\text { realizarla. }\end{array}$ & $\begin{array}{l}\text { Registros con } \\
\text { ideas } \\
\text { generales, } \\
\text { apenas } \\
\text { descriptivos; } \\
\text { muestras de } \\
\text { pocas } \\
\text { evidencias; } \\
\text { asomo de } \\
\text { alguna } \\
\text { oportunidad de } \\
\text { mejora o } \\
\text { crecimiento; } \\
\text { reflexiones } \\
\text { superficiales, } \\
\text { con potencial } \\
\text { de crecimiento. }\end{array}$ & $\begin{array}{l}\text { Registros } \\
\text { pobres, poco } \\
\text { claros; no hay } \\
\text { descripción de } \\
\text { todas las } \\
\text { actividades, } \\
\text { solo de } \\
\text { algunas; hay } \\
\text { carencia de } \\
\text { muestras de } \\
\text { evidencias, } \\
\text { posiblemente } \\
\text { algunas pero } \\
\text { poco claras; la } \\
\text { reflexión es } \\
\text { muy superficial } \\
\text { o está ausente, } \\
\text { así como las } \\
\text { ideas de } \\
\text { mejora. }\end{array}$ & $\begin{array}{l}\text { No realizó la } \\
\text { bitácora. No } \\
\text { existe } \\
\text { justificación } \\
\text { válida para } \\
\text { la falta de } \\
\text { trabajo. }\end{array}$ \\
\hline 2. Otros... & & & & & & \\
\hline $\begin{array}{l}\text { Puntaje } \\
\text { total por } \\
\text { nivel: }\end{array}$ & & & & & & \\
\hline
\end{tabular}

Fuente: elaboración propia: Gabriela Murillo S. 
Tanto para valorar cada uno de los niveles de desempeño anteriores, así como para considerar la suma total de la matriz, se consigna como ejemplo la siguiente interpretación:

\begin{tabular}{|c|c|}
\hline Calificación & Descripción general (se amplía según lo que se requiera) \\
\hline $\begin{array}{l}\text { Creativo/ } \\
\text { propositivo }\end{array}$ & $\begin{array}{l}\text { Nivel de desempeño excepcional. Realiza propuestas estratégicas. Excede lo esperado. Muestra } \\
\text { comprensión de gran nivel sobre las problemáticas y situaciones analizadas. Se evidencia } \\
\text { creatividad. Todos los procesos requeridos están incluidos en su desempeño. Muestra alto } \\
\text { compromiso con el trabajo, consigo mismo, con los demás. }\end{array}$ \\
\hline Propositivo & $\begin{array}{l}\text { Nivel de desempeño que supera lo esperado. No requiere asesoría ni supervisión; posee } \\
\text { argumentaciones de peso, fundamentadas. Sus propuestas son pertinentes, muestran } \\
\text { autonomía, son creativas. La comprensión de la realidad es considerable. La mayoría de los } \\
\text { procesos requeridos están incluidos en sus desempeños. El grado de compromiso con el trabajo, } \\
\text { consigo mismo y con los demás es notable. }\end{array}$ \\
\hline Autónomo & $\begin{array}{l}\text { Nivel de desempeño superior al estándar. Requiere cierta asesoría y seguimiento. Posee } \\
\text { argumentaciones de peso, con cierta fundamentación. Sus propuestas son pertinentes, en } \\
\text { ocasiones creativas. La comprensión del problema es suficiente. La mayoría de los procesos } \\
\text { requeridos están incluidos en sus desempeños. Autonomía presente en general. El grado de } \\
\text { compromiso con el trabajo, consigo mismo y con los demás es aceptable. }\end{array}$ \\
\hline Suficiente & $\begin{array}{l}\text { Nivel de desempeño estándar. Requiere mucha asesoría y seguimiento; sus argumentaciones se } \\
\text { muestran muy poco o nada fundamentadas. Sus propuestas no resultan suficientemente } \\
\text { pertinentes y la creatividad apenas se vislumbra. La comprensión del problema es apenas } \\
\text { razonable. Muchos de los procesos requeridos están incluidos en sus desempeños. Baja } \\
\text { autonomía. El grado de compromiso con el trabajo, consigo mismo y con los demás es apenas } \\
\text { aceptable. }\end{array}$ \\
\hline Deficiente & $\begin{array}{l}\text { Nivel de desempeño por debajo de lo esperado. Baja autonomía y también baja autoestima. } \\
\text { Ausencia de propuestas. Acciones que muestran nociones muy generales, sin organización. La } \\
\text { comprensión de la realidad es escasa. Hay muestra de conocimientos sin manejo de } \\
\text { procedimientos básicos, los cuales están ausentes en la mayoría de los procesos. Bajo nivel de } \\
\text { compromiso. }\end{array}$ \\
\hline No aceptable & $\begin{array}{l}\text { No satisface nada de lo esperado. No comprendió lo que debía realizar. Carece de autonomía. } \\
\text { No hizo el trabajo. }\end{array}$ \\
\hline
\end{tabular}

Fuente: elaboración propia: Gabriela Murillo S.

Pensando en los docentes y en algunas fuentes que sirvan como base para construir una matriz, se seleccionaron dos ejemplos que muestran criterios específicos que eventualmente pueden ser utilizados para su evaluación. Los modelos son: el caso de la Universidad de Costa Rica (Consejo Universitario, UCR, 2004) y el de la Universidad Interamericana de Puerto Rico (1998), ambos fueron escogidos con el fin de citar algunos rasgos que se esperaría sean observables en un docente, y por ende, reflejados en su propio portafolio. De la misma manera, cuando se trata de los estudiantes, al trabajar los aprendizajes desde competencias pueden establecerse perfiles cuyos rasgos sean observables en criterios concretizados en portafolios y sus rúbricas respectivas. Se destacan, entonces, los dos casos a manera de ejemplo: 
1. De acuerdo con el Perfil de Competencias Genéricas para el profesorado de la Universidad de Costa Rica, se esperan desempeños docentes tales como:

\section{Docencia}

- Conocimiento experto en su disciplina.

- Conocimiento de diversas perspectivas pedagógicas, metodologías y técnicas didácticas.

- Capacidad para orientar el proceso de enseñanza-aprendizaje de acuerdo con necesidades de aprendizaje del estudiantado.

\section{Investigación}

- Capacidad para analizar y resolver problemas.

- Capacidad para negociar y gestionar proyectos de investigación.

- Pensamiento crítico y reflexivo hacia sí mismo, los demás y el entorno.

\section{Acción Social}

- Conocimiento de la realidad nacional e internacional.

- Capacidad para trabajar en equipos interdisciplinarios.

- Capacidad para intercambiar conocimiento con las comunidades, personas y organizaciones.

\section{Personal}

- Ser flexible y adaptable a los cambios y las nuevas perspectivas e innovaciones en el conocimiento.

- Ser emprendedor, dinámico y crítico.

- Ser tolerante y respetuoso de los derechos de las otras personas sin discriminación de ningún tipo.

\section{Ético-institucional}

- Conocimiento y compromiso con la normativa institucional.

- Compromiso con los principios y funciones de la Institución.

- Fomentar los valores y principios éticos del humanismo. 
2. Como parte de las Guías para evaluación del personal docente, en la Universidad Interamericana de Puerto Rico se establecen criterios tales:

\section{Calidad docente}

- Dominio de la disciplina que enseña.

- Habilidad para organizar el contenido y presentarlo en forma clara, lógica e imaginativa.

- Conocimiento de los desarrollos actuales en la propia disciplina.

- Habilidad para relacionar su disciplina con otras esferas de conocimiento.

- Habilidad para promover y ampliar el interés del estudiante en la asignatura.

- Habilidad para desarrollar y utilizar métodos y estrategias adecuados, incluyendo el uso de la tecnología educativa, para una enseñanza efectiva.

\section{Servicio a la Institución}

- Trabajo en comités de facultad, de escuela o de departamentos.

- Participación y aportación a reuniones de facultad y de comités en el nivel de la unidad y otros.

- Servicio en comités y en organizaciones institucionales y estudiantiles.

- Colaboración con actividades estudiantiles.

- Asignaciones especiales.

- Designaciones como directorla de departamento, presidentela de comité y otras.

\section{Investigación y trabajo creativo}

- Publicaciones.

- Investigaciones no publicadas, ponencias o monografías relacionadas con sus desempeños o con la disciplina.

- Concesión de ayudas para investigación y proyectos.

- Invenciones y patentes.

- Labor artística y actuación.

\section{Reconocimiento profesional y actividades}

- Otorgamiento de premios, ayudas o becas.

- Nombramiento en capacidad asesora o ejecutiva en agencias del gobierno y privadas. 
- Participación activa en organizaciones profesionales.

- Recurso en conferencias y charlas.

- Participación en conferencias, congresos o institutos.

\section{Servicio a la comunidad}

- Servicio en el campo profesional del individuo como consultor o como investigador.

- Conferenciante de grupos de la comunidad.

- Participación activa en gestiones sociales, políticas, ambientales o de otro tipo.

\section{Fase 4. Publicación del portafolio}

De acuerdo con el diseño inicial o generando uno nuevo, se hace la publicación del portafolio. Esta publicación puede realizarse por etapas -avances programados- incluyendo una presentación final, o solamente una exposición de cierre del proceso. Entonces, en lo relativo al qué y al cuándo se publica, se trata de una fase que no constituye solamente una acción final, sino que puede incluir presentaciones en distintos momentos, según se consideren los avances en el desarrollo del portafolio.

La presentación final, particularmente, puede considerar el portafolio completo, o algunas de sus fases o evidencias de cada una de sus partes. Lo recomendado es que se presente lo más completo posible. En el caso del e-portfolio, existe la posibilidad de dar permisos de ingreso a ciertas secciones o a todo completo, de manera que siempre quedará a criterio de la persona que realiza el portafolio, reservarse alguna información si así lo decide.

En cuanto al para quién se publica, lo que tiene que ver con la persona o instancia que haya solicitado eventualmente presentación del portafolio, según las experiencias lo más frecuente es que el docente lo haga para otros pares y con el fin de algún reconocimiento en su carrera profesional, y el estudiante lo haga para el docente que lo ha requerido como parte del proceso evaluativo de su actividad académica o curso. En el caso de otro tipo de actividad o circunstancia, pues será para las personas que organizan y para otros involucrados.

Resulta relevante mencionar que lo importante de esta fase está en dar a conocer el proceso realizado, los desempeños alcanzados y, en la medida de lo posible, sus niveles de logro. Como un todo constituye una constancia de aprendizaje, de evolución, de compromiso 
por el mejoramiento continuo de la propia condición, como docente o como estudiante. Debe presentar coherencia interna y creatividad en la organización del producto final.

Dado lo novedoso que resulta respecto a la práctica común en cuanto a confección de portafolios, y con base en la propia experiencia, se destaca en este escrito el portafolio electrónico o e-portfolio. El siguiente modelo presenta con mucha claridad lo que constituye este tipo de portafolio:

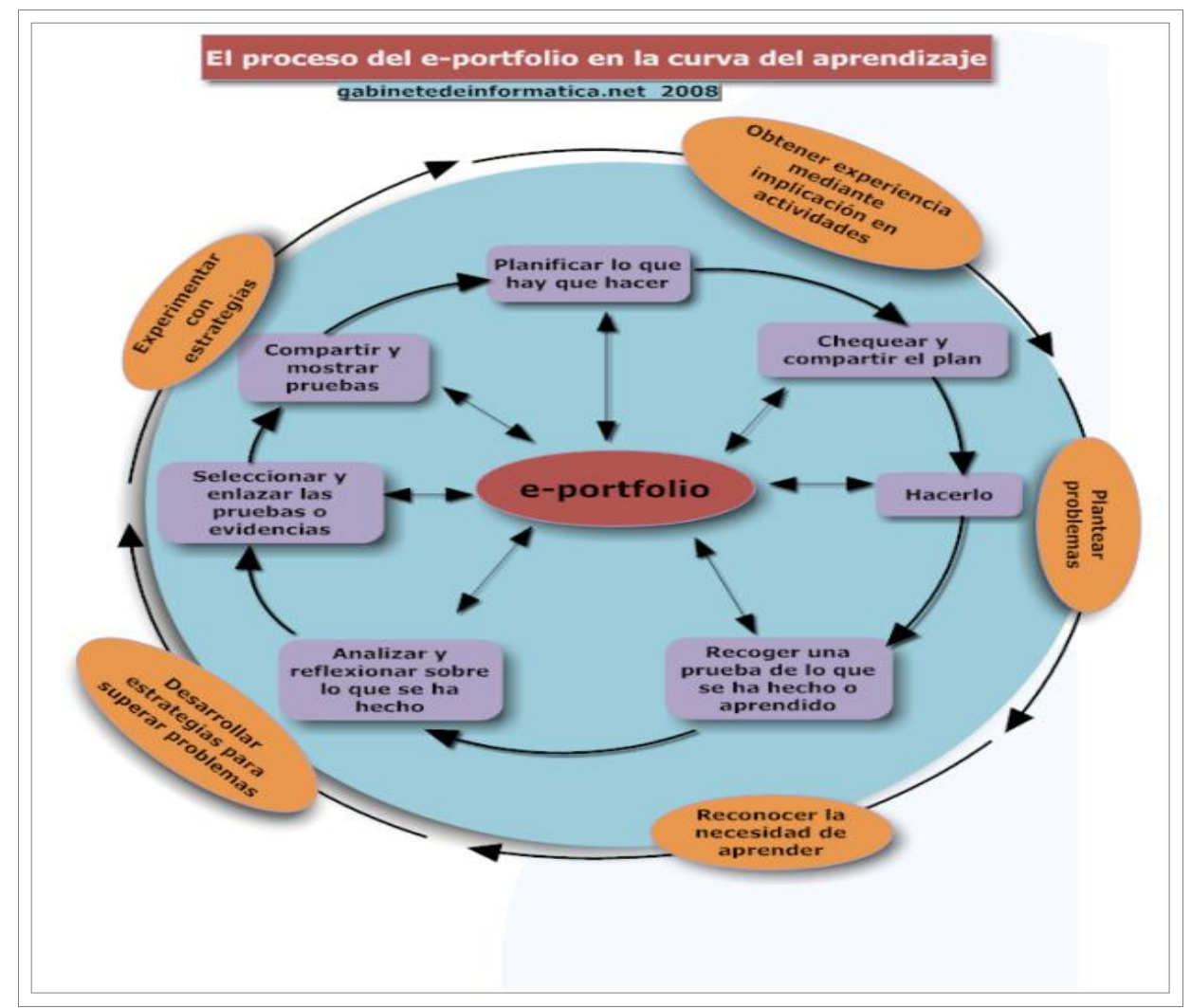

Fuente: Imagen de Fernando Santamaría publicada en Apuntes sobre e-portafolios

De acuerdo con Barret, (2000), su naturaleza gráfica y habilidad para soportar enlaces entre distintas evidencias digitalizadas, favorece la integración de los aprendizajes de un modo positivo, progresivo y consciente con un gran potencial atractivo. Constituye un sistema de gestión que permite a estudiantes, profesores y administradores la creación y organización de sus avances, documentos, resultados, producciones, en fin, lo que se genere durante el proceso de construcción del portafolio. 
A diferencia de un portafolio realizado en un formato impreso, la riqueza de las producciones en cuando a la diversificación de sentido es aún mayor, dado el lenguaje multimedia que ha de manejar. El hipertexto se utiliza para mostrar más claramente las relaciones entre objetivos, contenidos, procesos y reflexiones. A este punto es importante hacer una diferencia entre el e-portfolio y el portafolio digital, dado el dinamismo de los medios analógicos que emplea, la integración de tecnologías en su construcción.

Algunas posibilidades de realización del e-portfolio están dadas por los siguientes escenarios electrónicos:

$\checkmark$ Evernote: se trata de una herramienta sencilla, básica para experimentar la construcción de un e-portfolio.

http://www.evernote.com/

$\checkmark$ Google Sites: constituye otro instrumento básico para elaborar e-portfolios.

https://www.google.com/accounts/ServiceLogin?continue=http://sites.google.com/site/si tes/\&continue1=http://sites.google.com/site/sites/\&continue2=http://sites.google.com/sit e/sites/\&service $=$ jotspot\&u

$\checkmark$ Blogs: es la herramienta más utilizada para confeccionar un e-portfolio, los estudiantes le dan un papel preponderante.

https://www.blogger.com/start

$\checkmark$ Wordpress: junto con el instrumento anterior, el Worpress es favorito de los estudiantes por su dinamismo y porque resulta amigable cuando se utiliza.

http://es.wordpress.org/

$\checkmark$ Webquest: para investigación especialmente y recomendado para docentes y estudiantes que realizan sus trabajos de graduación:

http://www.didacticatic.educacontic.es/curso/internet-herramientas-y-aplicacionesweb/webquest-investigacion-medianteinternethttp://perso.gratisweb.com/cois600/assessment/eportafolio.htm http://www.didacticatic.educacontic.es/sites/default/files/tree/170/es/ver/index.html 
$\checkmark$ Elgg: plataforma que se utiliza principalmente para trabajar en red http://elgg.org/index.php

$\checkmark$ Mahara: plataforma especializada para e-portfolios. http://mahara.org/

$\checkmark$ The open Source Portfolio initiative: http://www.theospi.org/ http://www.becta.org/postnuke/modules.php?op=modload\&name=News\&file=article\&si $\mathrm{d}=146$ \&mode=thread\&order $=0$ \&thold $=0$ Open Source And Open Source World: http://www.becta.org/postnuke/modules.php?op=modload\&name=News\&file=index\&cat $\underline{\mathrm{id}=\text { \& topic }=24}$

$\checkmark$ Iniciativa para el desarrollo de proyectos colaborativos de la Universidad de Minnesota: http://www.becta.org/postnuke/modules.php?op=modload\&name=News\&file=article\&si $\underline{d=146}$

$\checkmark$ Weblogs para ePortfolios:

http://www.mcli.dist.maricopa.edu/ocotillo/eport/blog.php

$\checkmark$ Esta herramienta incluye la utilización de mapas conceptuales: http://compendium.open.ac.uk/institute/

\section{Discusión y conclusiones}

Si la evaluación es un recurso importante para el diagnóstico, para realizar transformaciones cuando se requieren, para el mejoramiento como una acción continua y final, ¿cómo desarrollarla?, ¿cómo impulsarla?, ¿cómo darle seguimiento? Entre diversos métodos, técnicas e instrumentos, ¿será entonces el portafolio un instrumento que favorezca la evaluación con una intención articuladora, reflexiva, de impulso hacia el mejoramiento?

Es importante tener en cuenta que cuando una evaluación resulta efectiva y justa, necesariamente ha tomado en cuenta asuntos tales como:

a) Criterios de evaluación diversos como marco de referencia, es decir, los principios para valorar la ejecución de las funciones y desempeños esperados 
por la persona.

b) Fuentes de información que evidencien el cumplimiento de los criterios de evaluación.

c) Funciones claras respecto de las personas que participarán en dicha evaluación.

d) Garantías sobre el proceso, tales como: completo conocimiento por parte de la persona evaluada de la información y los criterios considerados y requeridos para su evaluación; oportunidad para suministrar información adicional, probatoria.

e) Devolución de resultados en distintos momentos de la evaluación, según se requiera. Esto con el fin de que la persona evaluada pueda realizar el mejoramiento necesario y sepa también cuáles son los resultados intermedios y/o finales.

En el caso de la evaluación docente, particularmente, es importante conformar comisiones de evaluadores, incluir la participación de pares externos e incluir diversos elementos que favorezcan la integralidad del proceso: valoración de estudiantes, de jefaturas, y la suya propia (autoevaluación). Los instrumentos que se utilicen también son variados y según la experiencia internacional, la observación de clases, la respuesta a cuestionarios y/o entrevistas, la grabación de clases y el portafolio, son algunos de esos que, combinados, responden a una evaluación más justa, equitativa y pertinente.

Recapitulando desde lo básico hacia lo complejo, cuando nos referimos a un portafolio estamos considerando la colección de documentos del trabajo del docente o del estudiante, que muestra su esfuerzo, progreso y logros. Se trata de una forma de evaluación que le permite a alguien (docente en el caso de los estudiantes y pares en caso del docente) monitorear el proceso de aprendizaje y que, además, favorece el introducir cambios durante dicho proceso.

Es de esperarse que la evaluación refleje, entre otras cosas, la toma de decisiones que realiza la persona de manera procesual y que se muestra en una herramienta como el portafolio. Para ello, es importante tomar en cuenta, según Salas (2011, 凤 4 y 5), que tanto el pensamiento estratégico como las emociones tienen un rol fundamental en la toma de decisiones 
La capacidad humana de tener recuerdos del futuro, es decir, de imaginar diversos escenarios posibles y de evaluarlos racionalmente con base en la marca emocional que provocaron en el pasado, nos permite tomar decisiones sobre cuál es la mejor acción a seguir. Si el pensamiento es estratégico y funciona con base en la evaluación racional y emocional de escenarios o cursos de acción posibles, entonces, la solución de problemas o enigmas podría seguir una ruta similar.

Es fundamental reconocer al portafolio como un medio, no como un fin. Con esta herramienta se pretende recopilar la información que demuestre las habilidades y logros de quienes lo realizan, su manera de pensar, de cuestionarse y cuestionar, de analizar y analizarse, de sintetizar, de producir, de crear, y también de cómo interactuar (intelectual, emocional y socialmente) con otros, esto permite identificar rasgos de aprendizajes a nivel conceptual, procedimental y actitudinal de cada persona. Puede utilizarse en forma de evaluación, co-evaluación y/o autoevaluación (Argudín, 2007).

Para su construcción se recomienda a nivel general y ya sea por delimitación externa o por acuerdo: determinar los objetivos; seleccionar el contenido y la estructura; decidir cómo se va a manejar y presentar; establecer el tiempo para su desarrollo y también para su evaluación; comunicar apreciaciones y resultados parciales y finales; construir y aplicar la rúbrica evaluativa y su interpretación correspondiente, según la naturaleza del portafolio: docente o estudiantil.

Para establecer la finalidad y los objetivos del portafolio es importante plantearse preguntas tales como: ¿qué quiero lograr haciendo el portafolio?, ¿qué debe incluir?, ¿cuándo debo comenzarlo?, ¿cuándo terminarlo?, ¿cómo deberá ser presentado?, ¿qué deberá ubicarse en los anexos y qué como desarrollo central?, ¿cómo organizar su estructura?, ¿cuáles serán los aspectos por evaluar?, ¿cuáles los indicadores que tendrá cada aspecto?, ¿cuáles evidencias se presentarán en el portafolio?, ¿cómo se organizará su presentación de tal manera que muestren los avances?, ¿por qué resulta pertinente acompañar las evidencias con explicaciones sobre lo que son, el por qué se agregaron y de qué son evidencia?

Desde la perspectiva de quien evalúa un portafolio dar seguimiento a la organización y presentación de evidencias es fundamental, en término de los cambios y avances que éstas reflejan y de lo que ofrecen para poder observar, contrastar, sugerir, incentivar, preguntar y 
hasta modificar criterios e indicadores según se requiera. Las evidencias muestran rasgos de crecimiento, de desarrollo, de comprensión, de reflexión y análisis, de toma de decisiones y justificación de las mismas.

La elaboración de la rúbrica o matriz de evaluación y el delicado trabajo de la delimitación (y calificación) de los aspectos, criterios e indicadores, así como su puesta en práctica e interpretación, ponen en juego el alcance de la finalidad del portafolio, el mejoramiento de la persona que lo realiza. De ahí que resulte de suma importancia el rol de la persona evaluadora y sus propias competencias y desempeños en esta tarea.

Con todo esto se favorecería, entre otras cosas: la conciencia de estar siempre aprendiendo; la convicción de que las transformaciones son posibles y la importancia de realizar esfuerzos para fomentar y concretar innovaciones; el respeto por las personas (desarrolladores y evaluadores); la consecución de la creatividad y la autonomía; el enriquecimiento de la fundamentación con que se realizan las distintas acciones; la delimitación de elementos de juicio en la toma de decisiones y en la construcción y aplicación de una rúbrica de evaluación; la contextualización de las propias acciones; el fomento de actitudes y valores tales como la tolerancia, la honestidad, la responsabilidad y la amabilidad; estar disponible para el diálogo y saber escuchar; impulsar la defensa de los derechos y deberes propios; otros beneficios que tiendan a logros máximos en las distintas dimensiones de la formación humana de la persona que realiza el portafolio.

En esta concepción de portafolio en Educación Superior se requiere de una serie de competencias que reúnan rasgos específicos relacionados con lo que se va a proponer en la rúbrica de evaluación. De esta manera deberían considerarse competencias específicas requeridas para docentes (según rasgos presentados en el apartado anterior en los dos casos) y para el estudiantado. Se estaría suponiendo que dichas competencias tienen un soporte formativo para docentes y estudiantes y que de hecho el desarrollo del portafolio no solo las requeriría sino que estaría apoyando su formación. Se trata, entonces, de una acción formativa integral y articulada entre los elementos participantes: competencias, rasgos, saberes, criterios de desempeño, evidencias.

Cabe mencionar que la idea de competencia mencionada refiere a un concepto amplio y complejo de la misma, que no se circunscribe a acciones aisladas o a evidencias de medición únicamente, que tiene que ver con una cartografía compleja que explica y justifica por sí misma cada competencia. 
La valoración de los niveles de logro, que fueron delimitados para los desempeños esperados, requiere un proceso de seguimiento, de monitoreo, de enriquecimiento, en fin, tendiente a la búsqueda de mejoramiento, de identificación de dificultades, amenazas, fortalezas y oportunidades, lo que se denomina entonces como: proceso evaluativo.

Dado lo anterior, la realización de un portafolio constituye una alternativa ante las posibles limitantes en cuanto a técnicas y procedimientos para mejorar los aprendizajes docentes y estudiantiles. La mayor fortaleza del uso del portafolio es que convierte los desempeños, análisis y propuestas (acciones de mejora y otros), en el eje de las reflexiones. Permite aprender de ejemplos concretos las fortalezas y las debilidades de las propias acciones y el mejoramiento correspondiente.

Es por este motivo, y con base en la experiencia de quien escribe, que se recomienda como una de las mejores estrategias valorativas de desempeños (docentes) y evidencias (estudiantes) el uso del portafolio en una acción integrada: didáctica-evaluación.

\section{Referencias}

Argudín, María Luna. (2007). Evaluación/instrumentos centrados en el alumno. Portafolio. Recuperado el Diciembre de 2011, de http://hadoc.azc.uam.mx/evaluacion/portafolios.htm

Ament, Elizabeth, et al. (2003). E-PORTFOLIOS. Recuperado de http://www2.uwstout.edu/content/art/artedportfolios/aboutthis.html

Arbesú, María Isabel y Argumedo, Gabriela. (2010). El uso del portafolio como recurso para evaluar la docencia universitaria. Revista Iberoamericana de Evaluación Educativa, 3 (1e). Recuperado el 09 de Agosto de 2011, de: http://www.rinace.net/riee/numeros/vol3-num1 e/art10.pdf

Consejo Universitario, Universidad de Costa Rica. (2004). Acta No. 4932. Recuperado el 2009, de http://www.cu.ucr.ac.cr/actas/2004/4932.pdf

Delgado, Ana María y Olivier, Rafael. (abril, 2006). La evaluación continua en un nuevo escenario docente. Revista de Universidad y Sociedad del Conocimiento, 3 (1). Recuperado el 19 de 08 de 2011, de: http://www.uoc.edu/ojs/index.php/rusc/article/viewArticle/v3n1-delgado-oliver

Fernández, Amparo. (2004). El portafolio como estrategia formativa y de desarrollo profesional. Educar, 127-142. 
Fernández, Amparo y Maiqués, José María. (2011). La carpeta docente como herramienta de evaluación y de mejora de la calidad de la enseñanza. En Evaluación de políticas educativas: VIII Congreso Nacional de Teoría de la Educación (pp. 86-90). Huelva, España: Universidad de Huelva.

Freire, Paulo. (2006). Pedagogía de la autonomía: saberes necesarios para la práctica educativa. México: Siglo XXI Editores, S.A.

Generalitat Valenciana. (mayo, 2006). Consejería de Empresa, Universidad y Ciencia. Recuperado el Diciembre de 2011, de http://www.recursoseees.uji.es/fichas/fm4.pdf

Universidad Miguel Hernández, España. (mayo, 2006). El portafolio del estudiante. Recuperado el 08 de Agosto de 2011, de http://www.recursoseees.uji.es/fichas/fm4.pdf

Mercé, Gisbert. (febrero, 2002). El nuevo rol del profesor en entornos tecnológicos. Acción Pedagógica, 11 (1). Recuperado el 19 de Agosto de 2011, de http://www.comunidadandina.org/bda/docs/VE-EDU-0008.pdf

Salas, Flora. (05 de mayo de 2011). Temas de actualidad en docencia universitaria [Mensaje en un blog]. Recuperado el 19 de Agosto de 2011, de DEDUN-UCR: http://www.docenciauniversitaria.ucr.ac.cr/blog/?p=191

Santamaría, Fernando. (2008). El proceso del e-portafolio en la curva del aprendizaje [Imagen en un blog]. Recuperado en Agosto de 2011, de Apuntes sobre eportafolios: http://fernandosantamaria.com/blog/2008/04/apuntes-sobre-e-portafolios/

Universidad Interamericana, Puerto Rico. (1998). Guías para la evaluación del personal docente. Recuperado el 01 de Agosto de 2011, de http://ponce.inter.edu/nhp/contents/RecHumanos-GuiasEvalPersonalDocente.htm

Velásquez, Robert. (2007). Rúbrica. Recuperado el Agosto de 2011, de Universidad de San Martín de Porres: http://es.scribd.com/doc/2905226/Rubricas-de-evaluacion 